\title{
Lactobacillus rhamnosus GG Reduces $\beta$-conglycinin-Allergy- Induced Apoptotic Cells by Regulating Bacteroides and Bile Secretion Pathway in Intestinal Contents of BALB/c Mice
}

\author{
Xiaoxu Chen ${ }^{1}$, Yuekun $\mathrm{Wu}^{2}$, Yaozhong $\mathrm{Hu}^{2}$, Yan Zhang ${ }^{2, *}$ and Shuo Wang ${ }^{2}$ \\ 1 Key Laboratory of Food Nutrition and Safety, Ministry of Education, Tianjin University of Science and \\ Technology, Tianjin 300457, China; chenxiaoxu_@126.com \\ 2 Tianjin Key Laboratory of Food Science and Health, School of Medicine, Nankai University, Tianjin 300071, \\ China; yuekunw@126.com (Y.W.); yzhu@nankai.edu.cn (Y.H.); wangshuo@nankai.edu.cn (S.W.) \\ * Correspondence: yzhang@nankai.edu.cn; Tel.: +86-22-85358445
}

Citation: Chen, X.; Wu, Y.; Hu, Y.; Zhang, Y.; Wang, S. Lactobacillus

rhamnosus GG Reduces

$\beta$-conglycinin-Allergy-Induced Apoptotic Cells by Regulating Bacteroides and Bile Secretion Pathway in Intestinal Contents of BALB/c Mice. Nutrients 2021, 13, 55. https:// dx.doi.org/10.3390/nu13010055

Received: 1 December 2020 Accepted: 24 December 2020 Published: 27 December 2020

Publisher's Note: MDPI stays neutral with regard to jurisdictional claims in published maps and institutional affiliations.

Copyright: $\odot 2020$ by the authors. Licensee MDPI, Basel, Switzerland. This article is an open access article distributed under the terms and conditions of the Creative Commons Attribution (CC BY) license (https://creativecommons.org/ licenses/by/4.0/).

\begin{abstract}
Allergy can cause intestinal damage, including through cell apoptosis. In this study, intestinal cell apoptosis was first observed in the $\beta$-conglycinin $(\beta-C G)$ allergy model, and the effect of Lactobacillus rhamnosus GG (LGG) on reducing apoptosis of cells in the intestine and its underlying mechanisms were further investigated. Allergic mice received oral LGG daily, and intestinal tissue apoptotic cells, gut microbiota, and metabolites were evaluated six and nine days after intervention. Terminal deoxynucleotidyl transferase-mediated dUTP nick-end labeling (TUNEL) analysis revealed that LGG intervention could reduce the incidence of cell apoptosis more effectively than natural recovery (NR). The results of $16 \mathrm{~S}$ rRNA analysis indicated that LGG intervention led to an increase in the relative abundance of Bacteroides. Metabolite analysis of intestinal contents indicated that histamine, $N$-acetylhistamine, $N(\alpha)$ - $\gamma$-glutamylhistamine, phenylalanine, tryptophan, arachidonic acid malate, and xanthine were significantly decreased, and deoxycholic acid, lithocholic acid were significantly increased after the LGG intervention on $\beta$-CG allergy; the decreases in histamine and $N(\alpha)$ - $\gamma$-glutamylhistamine were significant compared with those of NR. In conclusion, LGG reduces apoptosis of cells induced by $\beta-C G$ allergy, which may be related to regulation of Bacteroides and the bile secretion pathway.
\end{abstract}

Keywords: $\beta$-conglycinin; allergy; apoptotic cells; LGG; gut microbiota; metabolome

\section{Introduction}

$\beta$-Conglycinin ( $\beta-\mathrm{CG})$ is the main allergen of soybean. $\beta$-CG is a trimeric structure composed of $\alpha(67 \mathrm{kDa}), \alpha^{\prime}(71 \mathrm{kDa})$, and $\beta(50 \mathrm{kDa})$ subunits. Each subunit is composed of an acidic peptide chain and a basic peptide chain, and the structure is very stable and therefore persistent, resulting in relatively constant sensitization toward $\beta-C G[1,2]$. Allergies can cause disorders of the immune system and cell apoptosis. Similarly, allergies are accompanied by the appearance of a large number of inflammatory cells whose survival is regulated by apoptosis. Hence, an increase in apoptotic cells helps to accelerate the inflammatory process [3,4]. Apoptotic cells may also damage intestinal mucosal immunity [5]. At present, the main challenge in managing allergies is related to the lack of effective measures and potential mechanisms for reducing apoptotic cells.

Probiotics can prevent human intestinal diseases. When appropriately consumed, they produce a beneficial effect on the host through immune regulation. Some studies have confirmed that probiotics have immunomodulatory effects on allergies [6,7]. Probiotics can change the composition of gut microbiota and regulate immune response of the host as well as the intestinal mucosa through effects on antigens [8-10]. The potential of probiotics to alleviate allergic response lies in their ability to affect processes involved in the repair of the intestinal barrier function, which regulates balance in the imbalanced microbiome and, thereby, the immune system [11]. 
Gut microbiota are essential for healthy immune regulation and gut barrier function. The gut microbiota can interact with intestinal metabolites, such as bile acids (BAs), and regulate the host metabolism mainly by activating immune genes in the small intestine to regulate microbial composition in the body [12,13]. BAs regulate immune and inflammatory processes through signaling transduction, such as through the farnesoid $X$ receptor (FXR)regulated pathway and cell surface $G$ protein-induced signaling [14]. BAs can control the release of intestinal microbial immunoglobulin A (IgA) antibodies [15] and play an essential role in immune homeostasis of the intestine. BAs have anti-inflammatory effects on intestinal epithelial cells [16].

Lactobacillus rhamnosus GG (LGG) is one of the well-known commercialized probiotics. According to reports, LGG produces a preventive effect on the process of birch pollen allergic asthma [17]. LGG inhibits allergic inflammation in asthmatic mice [18] and alleviates allergic airway inflammation through gut microbiota [19]. LGG consumption results in a significant improvement in patients with milk protein allergy [20], and may alleviate allergy by improving intestinal homeostasis [21]. We previously verified that LGG can alleviate $\beta$-CG allergy by modulating immune gene expression in the T cell receptor (TCR) signaling pathway [22].

However, there is no published research on whether LGG can reduce cell apoptosis and whether the alleviating effect is related to the metabolism of the gut microbiota. In this work, the main purpose was to investigate the effect of LGG on $\beta$-CG-allergy-induced cell apoptosis as well as its related mechanism on the gut microbiota and related metabolic pathways.

\section{Materials and Methods}

\subsection{Allergen}

The separation and purification process of $\beta-C G$ was performed based on a previously reported method [23].

\section{2. $L G G$}

The activation, culture and gavage concentration of LGG referred to our previously reported method [22].

\subsection{Animals}

BALB/c mice (4-6 weeks) were used in this experiment. The experimental conditions were based on our previously reported method [22]. Animals experiments procedures were approved by the Institutional Animal Ethics Committee (IRM-DWLL-2020094).

\subsection{Experimental Design}

The saline group and the control group were supplemented with saline ( $300 \mu \mathrm{L} /$ mouse per week) and cholera toxin (CT; $300 \mu \mathrm{L} /$ mouse per week), respectively. The $\beta$-CG group was supplemented with $\beta-C G$ and CT for 5 weeks to induce allergy. Next, the allergic mice received oral LGG $\left(1 \times 10^{9} \mathrm{CFU} / 600 \mu \mathrm{L} /\right.$ mouse/day $)$, had a normal diet, and were divided into four equal groups. The LGG-1 group mice and the LGG-2 group mice were supplemented with LGG and were sacrificed on day 6 and 9, respectively. The NR-1 and the NR-2 group mice received a normal diet and were sacrificed on day 6 and 9, respectively.

After all the experimental mice were sacrificed, different parts of intestinal tissue were separately fixed in $4 \%$ paraformaldehyde for terminal deoxynucleotidyl transferasemediated dUTP nick-end labeling (TUNEL) analysis. The intestinal contents were obtained using a sterile cotton swab and immediately placed in a sterile cryotube until $16 \mathrm{~S}$ rRNA sequencing analysis. The remaining intestinal contents were collected and stored for metabolomics analysis. 


\subsection{Allergy Symptom Score}

After the fifth gavage, mice were observed for clinical symptoms, which were scored according to a previously reported method [22].

\subsection{Analysis of Immunoglobulin E (IgE) and Histamine (HIS) in Mice Serum}

The contents of IgE and HIS were determined by enzyme linked immunosorbent assay (ELISA), referring to the manufacturer's instructions (Nanjing Jiancheng Bioengineering Institute, Nanjing, China).

\subsection{Detection of Apoptotic Cells by TUNEL Assay}

The intestinal tissue $(4 \mu \mathrm{m})$ sections were deparaffined, rehydrated, and permeabilized with proteinase K. An In Situ Cell Death Detection Kit (Roche Diagnostics Ltd., Kanton Bern, Switzerland) was used for TUNEL detection based on detection of fluorescein isothiocyanate according to the manufacturer's instructions, and apoptotic cells were observed. The total number of cells was counted after counterstaining of sections. The apoptotic cells were regarded as TUNEL-positive cells if they demonstrated obvious apoptotic morphology. Five fields of view were randomly selected to analyze the samples under a microscope with a $40 \times$ objective.

\section{8. $16 S$ rRNA Gene Sequencing Analysis}

First, the DNA in the contents of the mouse intestine was extracted and sequenced using IonS5TMXL, and we conducted sequences analysis using Uparse software (Uparse v 7.0.1001, http://drive5.Com/uparse/). Taxonomic information of the representative sequence was annotated based on the Silva Database (https:/ /www.arb-silva.de/) through the Mothur algorithm. Multiple sequence alignments were finalized by utilizing the MUSCLE software (Version 3.8.31, http:/ / www.drive5.com/muscle/) to study phylogenetic relationship of different Operational Taxonomic Units (OTUs), as well as the difference of the dominant species in different groups. The data from OTUs clustering and species classification were analyzed. The related downstream analysis and data visualization were accomplished by QIIME (Version1.7.0) and displayed with R software (Version 2.15.3).

\subsection{Metabolomics Analysis}

Approximately $100 \mathrm{mg}$ of mouse intestinal contents were ground separately with liquid nitrogen and resuspended in methanol and formic acid. The samples were incubated on ice and centrifuged, and we adjusted the final concentration to a solution containing $60 \%$ methanol. The raw data files generated after UHPLC-MS/MS were processed by using the Compound Discoverer 3.0 (CD 3.0, Thermo Fisher) to realize peak alignment, peak picking, and quantitation of each metabolite. Finally, the predicted the molecular formula was compared using the mzCloud (https://www.mzcloud.org/) and Chemspider (http:/ / www.chemspider.com/) databases. KEGG pathway analysis was packaged based on Python software (Python-3.5.0).

\subsection{Statistical Analysis}

All data analyses were performed using SPSS 17.0 software (SPSS Inc, Chicago, IL, USA) and were expressed as mean \pm standard deviation. One-way ANOVA nonparametric testing was performed to determine statistical significance. $p<0.05$ was considered statistically significant.

\section{Results}

\section{1. $\beta$-CG Induced Intestinal Cell Apoptosis in Allergic Mice}

We first established the $\beta$-CG allergy model to observe the influence on intestinal apoptotic cells. The clinical symptoms of the mice administered oral saline and CT were normal. However, the allergic mice demonstrated significant clinical symptoms of allergy, such as diarrhea and erect hair (Figure 1A). IgE and HIS were significantly increased in 
the allergic mice (Figure 1B,C). Using the allergy symptom score and the typical allergy indicators IgE and HIS, we found that the $\beta$-CG allergy model was successfully established. The percentage of apoptotic cells in allergic mice was significantly higher than in the other groups (Figure 1D), indicating that $\beta-C G$ allergy induced cell apoptosis. The pathological status of the duodenum, jejunum, ileum, and colon was determined using TUNEL assay (Figure 1E-H). The intestinal villi were completely and neatly arranged, and no inflammatory cell infiltration was observed in the propria nor edema in the saline group. We observed that the thickness of the mucosa increased slightly, the membrane propria was loosened, there were a few inflammatory cells, and only a small part of the intestinal villi was damaged in the control group. Typical pathological features of intestinal allergy were observed in the intestinal tissues of allergic mice, such as a large number of ruptured intestinal villi, inflammatory cell infiltration, and intestinal mucosal edema. Therefore, the $\beta-C G$ allergy was determined to have caused cell apoptosis of the intestinal tissue based on the number of apoptotic cells and the pathological status of the intestinal tissue.
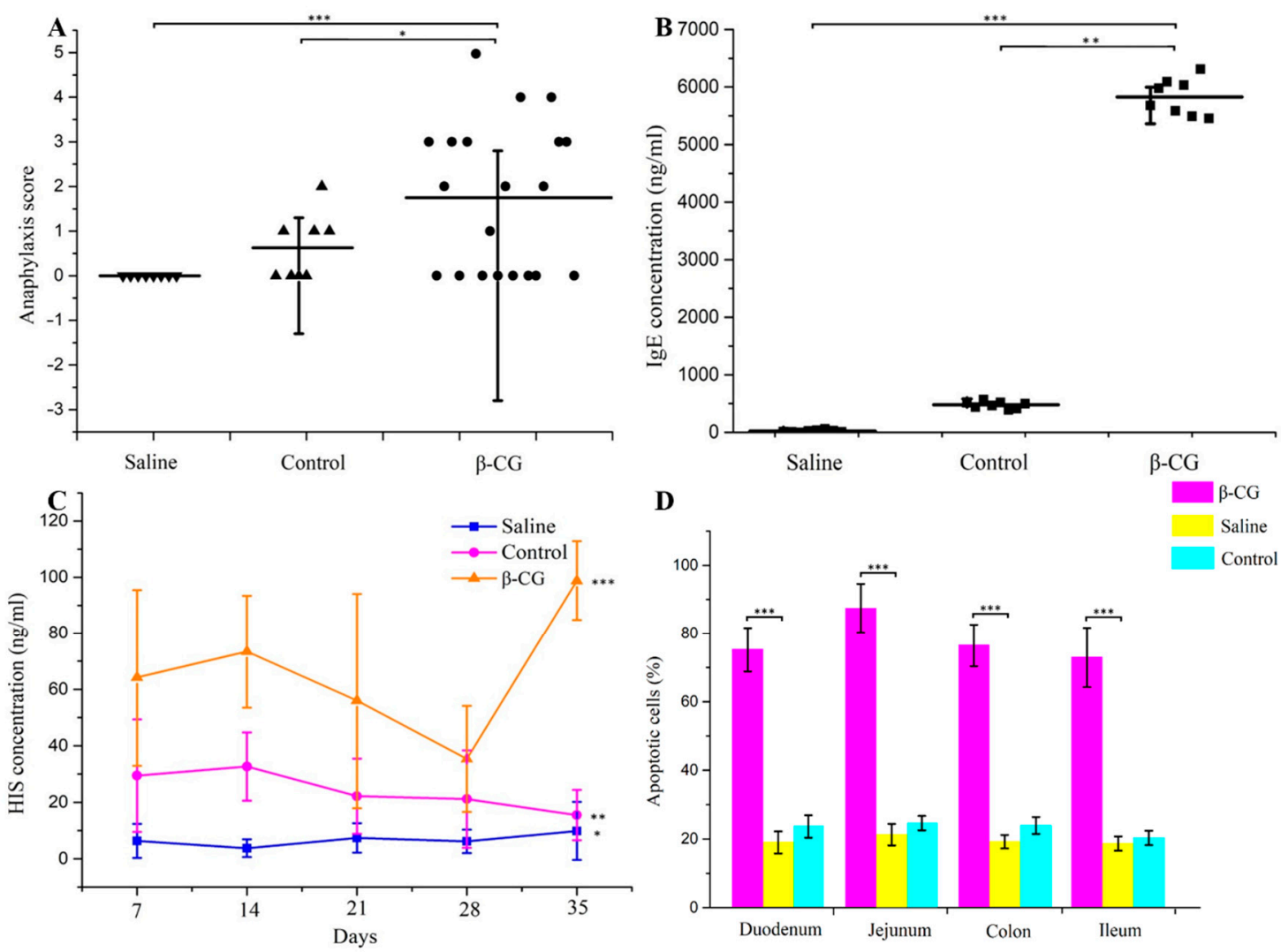

Figure 1. Cont. 

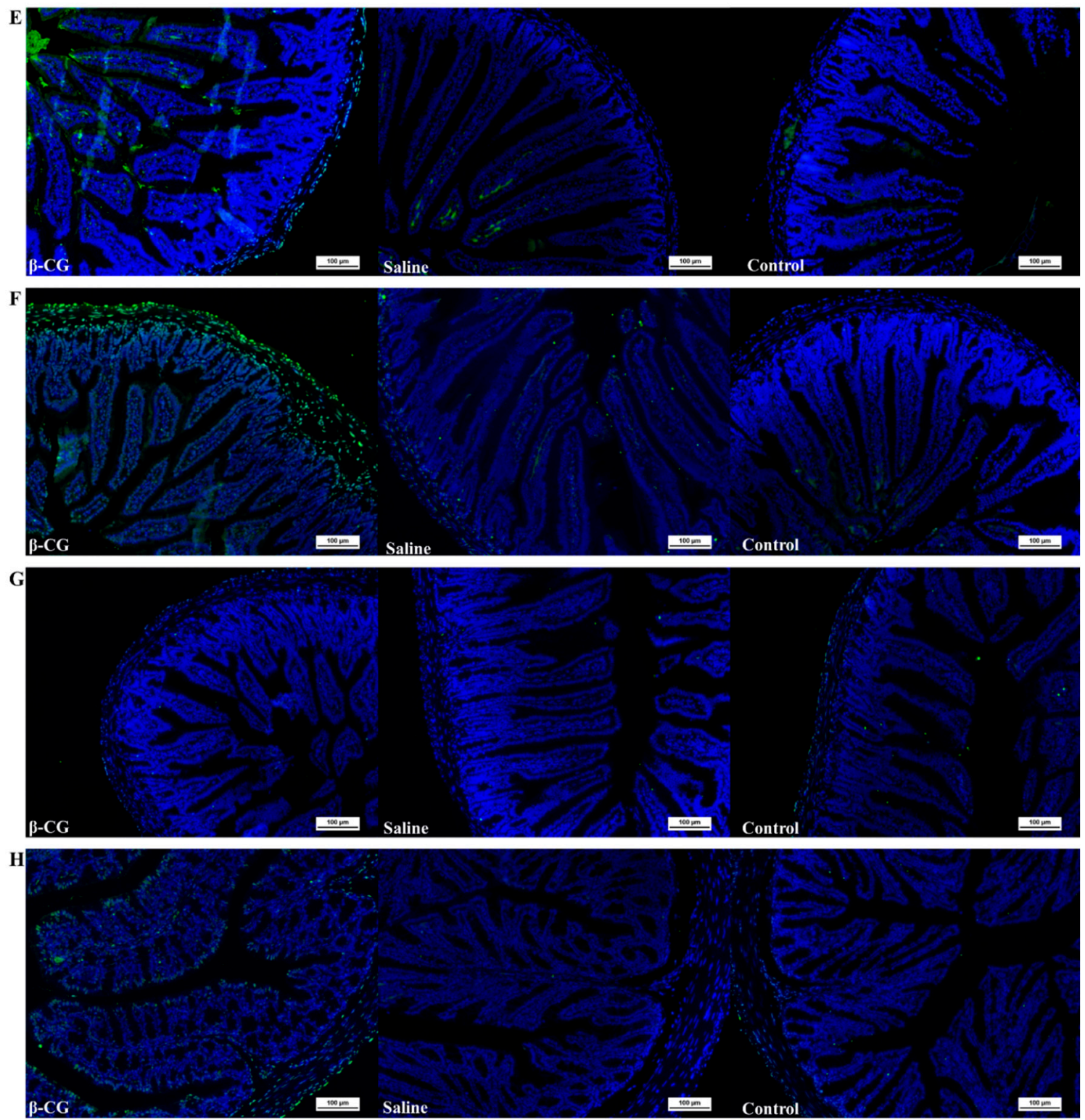

Figure 1. $\beta$-conglycinin ( $\beta-C G$ ) induced intestinal cell apoptosis in allergy mouse. (A) The score of anaphylaxis symptoms; (B) Levels of IgE; (C) Levels of histamine (HIS). (D) Terminal deoxynucleotidyl transferase-mediated dUTP nick-end labeling (TUNEL) assay on the number of apoptotic cells in duodenal, jejunum, ileum, colon. TUNEL assay on the pathological status in (E) duodenal; (F) jejunum; (G) ileum; (H) colon. ( ${ }^{*} p<0.05 ;{ }^{* *} p<0.01$; $\left.{ }^{* * *} p<0.001\right)$.

\subsection{LGG Reduces Intestinal Apoptotic Cells Induced by $\beta$-CG Allergy}

TUNEL assay was used to detect the number of apoptotic cells in the duodenum, jejunum, ileum, and colon (Figure 2A). The number of apoptotic cells were significantly reduced in the LGG-1 and LGG-2 groups. Compared with the NR group, the decrease in the percentage of apoptotic cells was more significant in the LGG group. The results indicated that LGG could effectively alleviate cell apoptosis induced by $\beta-C G$ allergy, and the effect was better than that of NR.

The TUNEL assay was also used to determine the pathological status of the different parts of the intestinal tissue, including the duodenum, jejunum, ileum, and colon (Figure 2B-E). In the LGG group, there was no massive shedding of intestinal villi. The arrangement of intestinal villi and the thickness of the mucosal muscle layer returned to normal levels. The same pattern of apoptosis was observed in other parts of the intestine. 
The results showed that LGG intervention reduced the number of apoptotic cells and restored intestinal damage.
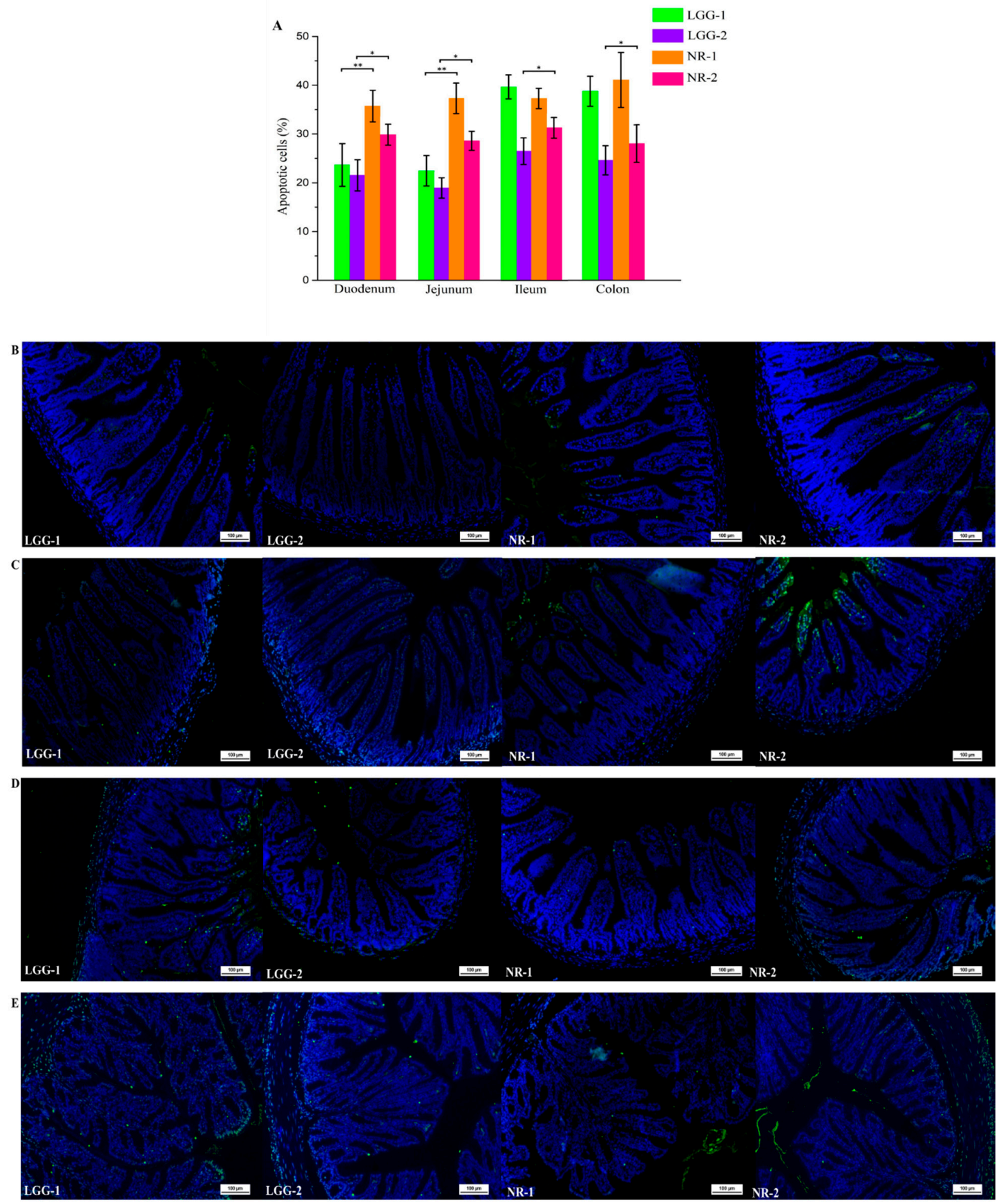

Figure 2. (A) TUNEL assay on the number of apoptotic cells in duodenal, jejunum, ileum and colon. TUNEL assay on the pathological status in (B) duodenal; (C) jejunum; (D) ileum; (E) colon. $\left({ }^{*} p<0.05\right.$; ${ }^{* *} p<0.01$ ).

\subsection{LGG Regulates the Imbalance of Gut Microbiota Induced by $\beta$-CG Allergy}

The $16 \mathrm{~S}$ rRNA sequencing analysis revealed the composition of gut microbiota in the intestine at the genus level (Figure 3A). We found that in allergic mice, the abundance of Bacteroides decreased while Cerasibacillus and unidentified_Lachnospiraceae increased. 
By contrast, the abundance of Bacteroides increased, and Cerasibacillus and unidentified _Lachnospiraceae decreased in the LGG group. The heat map was clustered through levels of species and samples (Figure 3B) and showed that the abundance of Acidothermus, Cerasibacillus, Roseburia, and unidentified_Lachnospiraceae increased after stimulation with $\beta$-CG. After LGG intervention, the abundance of Roseburia and unidentified_Lachnospiraceae decreased. In summary, these results indicated that $\beta-C G$ allergy induced imbalance in gut microbiota and that LGG intervention could regulate this imbalance.

A

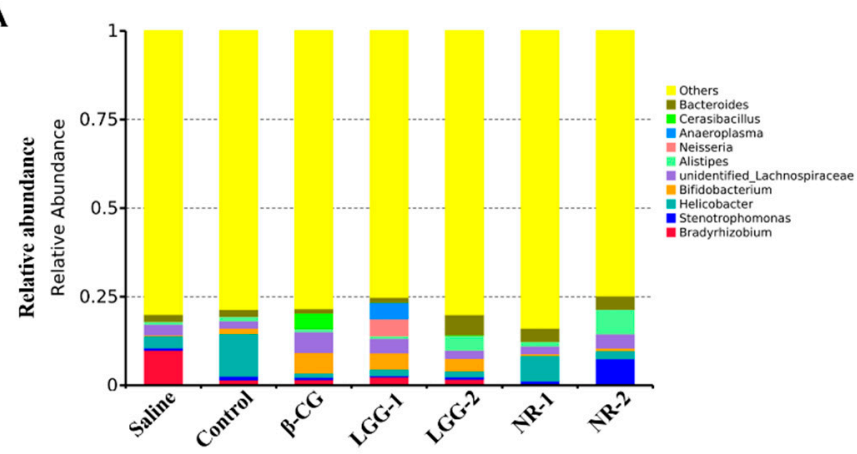

B

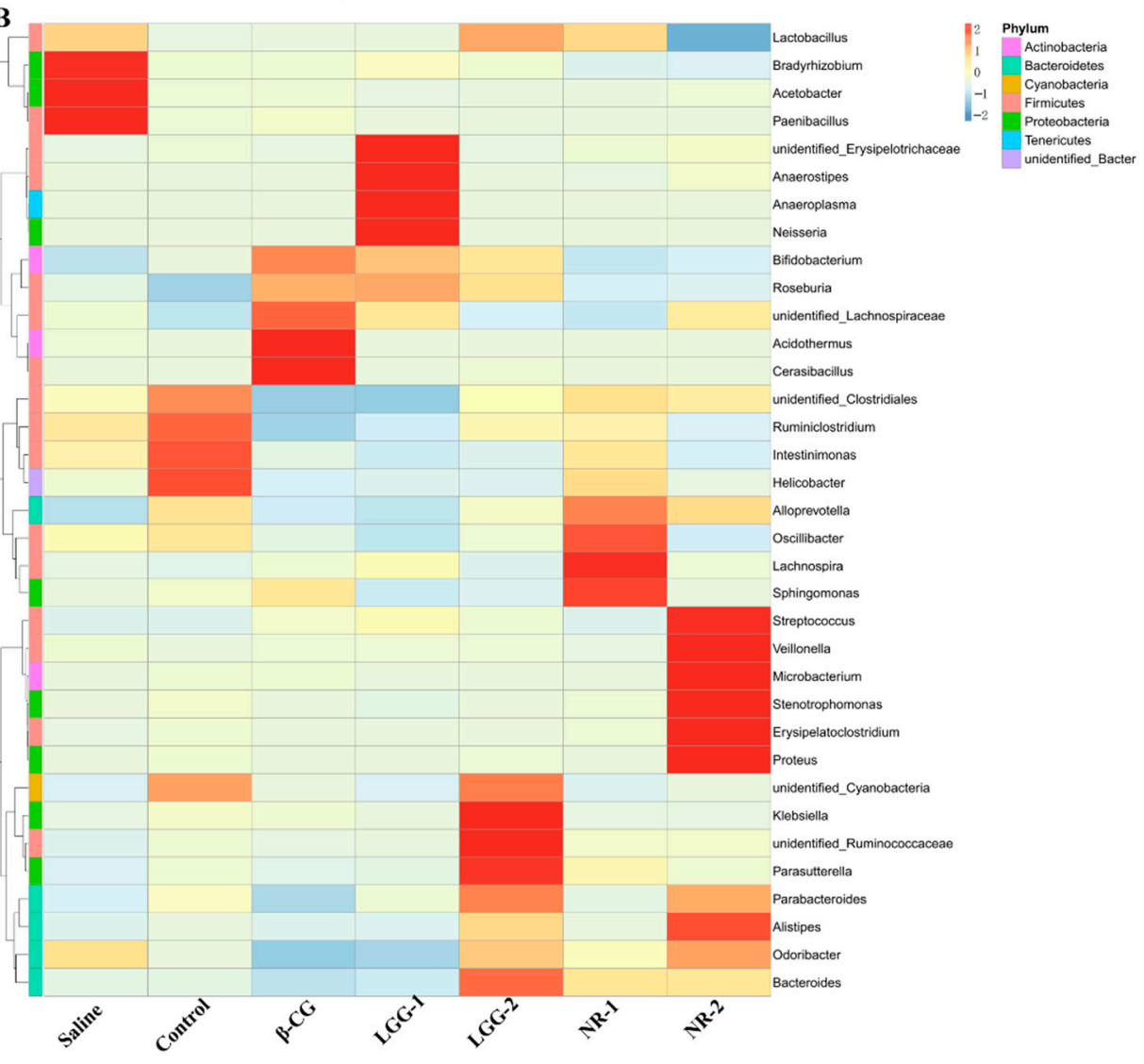

Figure 3. The genus level analysis of gut microbiota in intestinal contents. (A) Histogram of relative abundance of gut microbiota; (B) Heat map of gut microbiota.

\subsection{LGG Alleviates Intestinal Metabolites Disorder Induced by $\beta$-CG Allergy}

The metabolic profiles of the intestinal contents were analyzed by LC-MS/MS in positive and negative modes (Figure 4A-D). The clear separation between the LGG-1 and NR-1, and the LGG-2 and $\beta$-CG groups was obtained by Partial Least Squares Discriminant Analysis (PLS-DA), indicating that LGG intervention significantly affected metabolites. The LGG-1 and NR-1 groups were compared in positive $\left(R^{2}=0.99\right.$ and $\left.Q^{2}=0.91\right)$ and negative 
$\left(R^{2}=0.99\right.$ and $\left.Q^{2}=0.91\right)$ ion modes. The LGG-2 and $\beta$-CG groups were compared, with $\mathrm{R}^{2}=0.99$ and $\mathrm{Q}^{2}=0.64$ in positive ion mode, and $\mathrm{R}^{2}=0.99$ and $\mathrm{Q}^{2}=0.54$ in negative ion mode. The results showed that the model had good interpretation and prediction ratios.

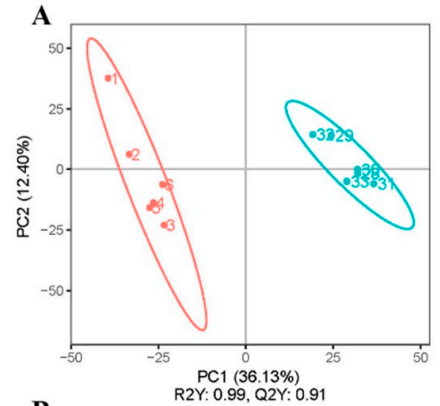

B
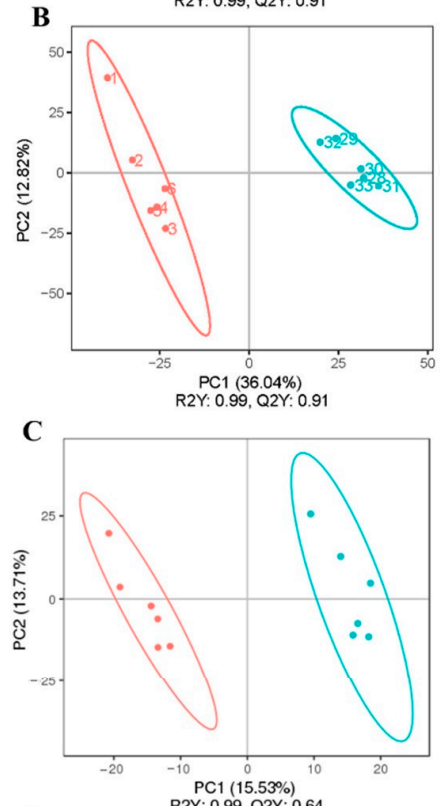

D

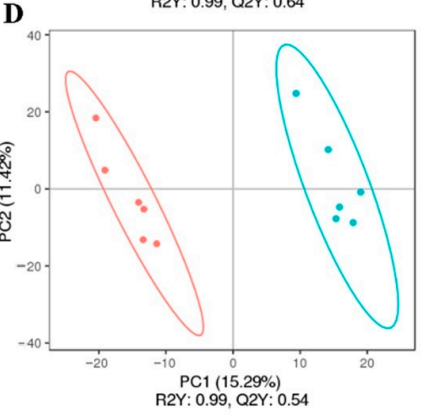

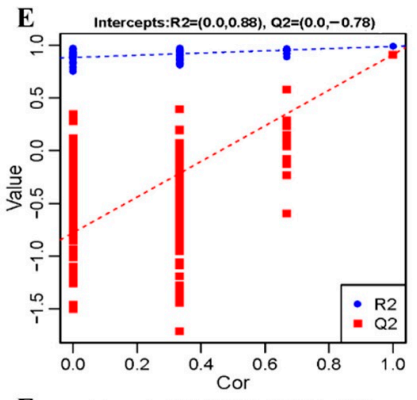
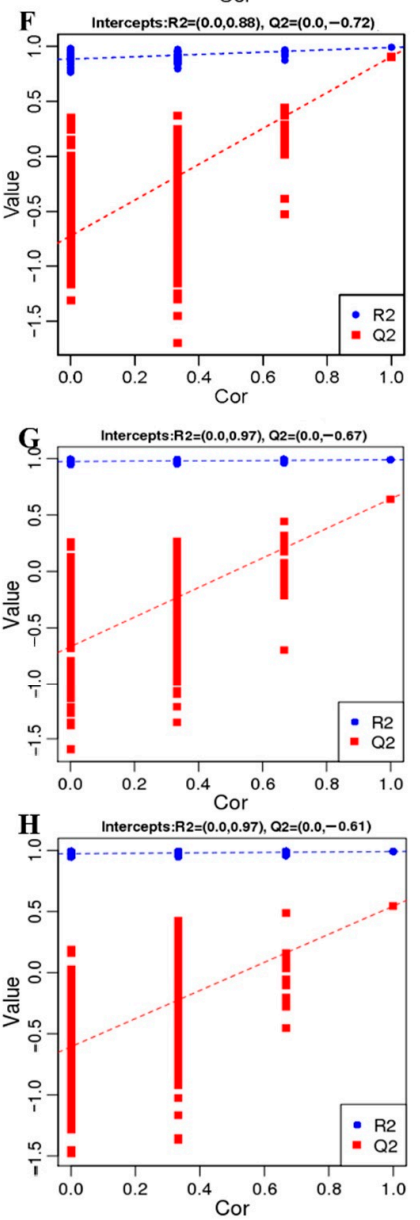

Figure 4. Partial Least Squares Discriminant Analysis (PLS-DA) analysis of intestinal contents. (A) PLS-DA scores plots depicting obvious difference between the Lactobacillus rhamnosus GG (LGG)1 group and the natural recovery (NR)-1 group in positive ion mode. (B) PLS-DA scores plots depicting obvious difference between the LGG-1 group and the NR-1 group in negative ion mode. (C) PLS-DA scores plots depicting obvious difference between the LGG-2 group and the $\beta$-CG group in positive ion mode. (D) PLS-DA scores plots depicting obvious difference between the LGG-2 group and the $\beta$-CG group in negative ion mode. (E) Sorting verification chart depicting obvious difference between the LGG-1 group and the NR-1 group in positive ion mode. (F) Sorting verification chart depicting obvious difference between the LGG-1 group and the NR-1 group in negative ion mode. (G) Sorting verification chart depicting obvious difference between the LGG-2 group and the $\beta$-CG group in positive ion mode. $(\mathbf{H})$ Sorting verification chart depicting obvious difference between the LGG-2 group and the $\beta-C G$ group in negative ion mode. 
The sorting test demonstrated the correlations between the LGG-1 group and the NR-1 group, and between the LGG-2 group and the $\beta$-CG group, which were $R^{2}(0.0,0.88)$ and $\mathrm{Q}^{2}(0.0,-0.78)$ in positive ion mode, $\mathrm{R}^{2}(0.0,0.88)$ and $\mathrm{Q}^{2}(0.0,-0.72)$ in negative ion mode, and $R^{2}(0.0,0.97)$ and $Q^{2}(0.0,0.67)$ in positive ion mode, and $R^{2}(0.0,0.97)$ and $Q^{2}(0.0$, 0.61 ) in negative ion mode, respectively (Figure $4 \mathrm{E}-\mathrm{H})$. The results showed that the model was not over-fitted and, thus, could describe the sample well and be used in the search for differential metabolite.

Hierarchical clustering analysis was performed on the differential metabolites in which the same or similar metabolic patterns were clustered and used to infer the function of unknown or known metabolites. Compared with the NR-1 group, we found obvious metabolic differences in positive ion mode in the LGG-1 group (Figure 5A). Figure 5B showed the comparison of differential metabolites of the LGG-2 and $\beta-C G$ groups in positive ion mode. Note areas with different colors, representing different clustering information; the results revealed that the mice subject to LGG intervention and $\beta-C G$ allergic mice used different metabolic pathways and, hence, had different metabolites in their intestine.

Figure 5C,D shows the correlation graph of differential metabolites in positive ion mode. Differential metabolites have a synergistic or mutually exclusive relationship. We analyzed the correlation between each metabolite by calculating Pearson correlation coefficients between all metabolites. When the linear relationship between the two metabolites increases, the positive correlation tends to 1 , and the negative correlation tends to -1 . The above results revealed that when comparing the LGG-1 group with the NR-1 group, Com_10093_pos was positively correlated with Com_10177_pos, Com_10402_pos, Com_10750_pos, and Com_10008_pos; Com_10297_pos was positively correlated with Com_10240_pos; Com_1007_pos was negatively correlated with Com_10001_pos; Com_10428_pos was negatively correlated with Com_10093_pos and Com_10177_pos. In comparing the LGG-2 and $\beta$-CG groups, Com_11020_pos was positively correlated with Com_12092_pos; Com_11284_pos was positively correlated with Com_10082_pos; Com_11102_pos was positively correlated with Com_12252_pos and negatively correlated with Com_10116_pos and Com_11859_pos.

The Z-score is a transformed value based on the relative content of differential metabolites in the mouse intestine and is used to compare the similarity of the differential metabolites. Figure 5E,F showed the relative metabolite contents of the first 30 compounds in the LGG-1, NR-1, LGG-2, and $\beta-C G$ groups. The Z-score analysis revealed the samples had good repeatability.

According to the above results, a bubble chart of the enriched Kyoto Encyclopedia of Genes and Genomes (KEGG) pathway was drawn. Compared with the NR-1 group, the main metabolic pathways in the LGG-1 group were the bile secretion pathway, histamine metabolism, and glycerophospholipid metabolism (Figure 6A). Compared with the $\beta$ CG group, the main metabolic pathway in the LGG-2 group was bile secretion pathway (Figure 6B). The results showed that the most differentially abundant metabolites after LGG intervention were of the bile secretion pathway.

Compared with the NR-1 group, histamine and $N(\alpha)$ - $\gamma$-glutamylhistamine were significantly downregulated in the LGG-1 group (Figure 7A). The results showed that allergic biomarkers were downregulated in the LGG group more than in the NR group, indicating that LGG intervention was more effective. Compared with the $\beta$-CG allergic mice (Figure 7B), histamine, $N(\alpha)$ - $\gamma$-glutamylhistamine, $N$-acetylhistamine, arachidonic acid, phenylalanine, tryptophan, malate, and xanthine were significantly downregulated in the LGG-2 group, whereas deoxycholic acid and lithocholic acid were significantly upregulated. The results showed that after LGG intervention, the levels of allergic biomarkers were significantly lowered. 
A

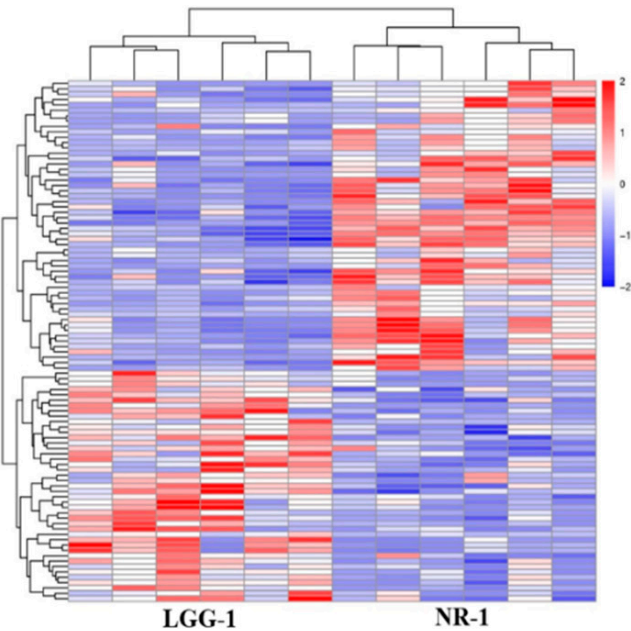

C

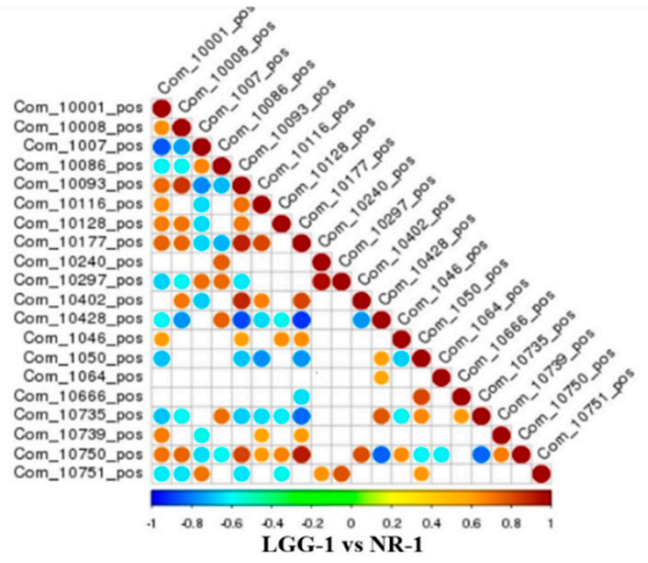

E

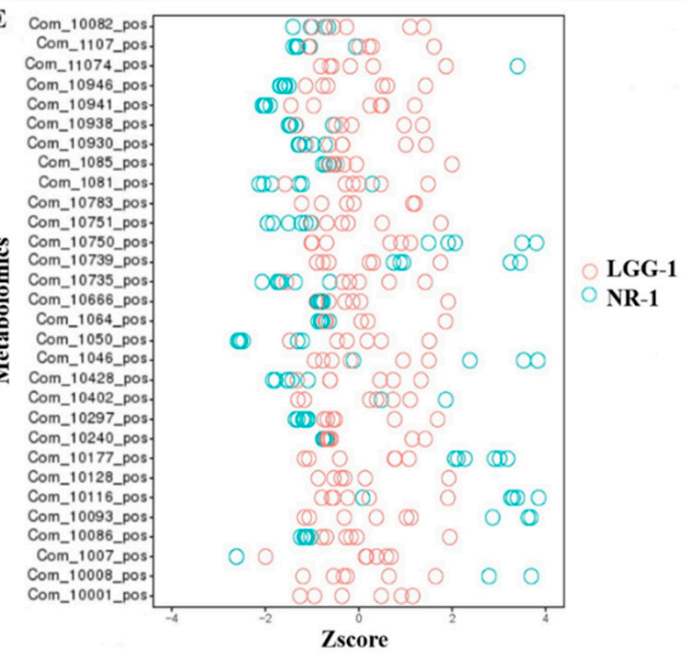

B

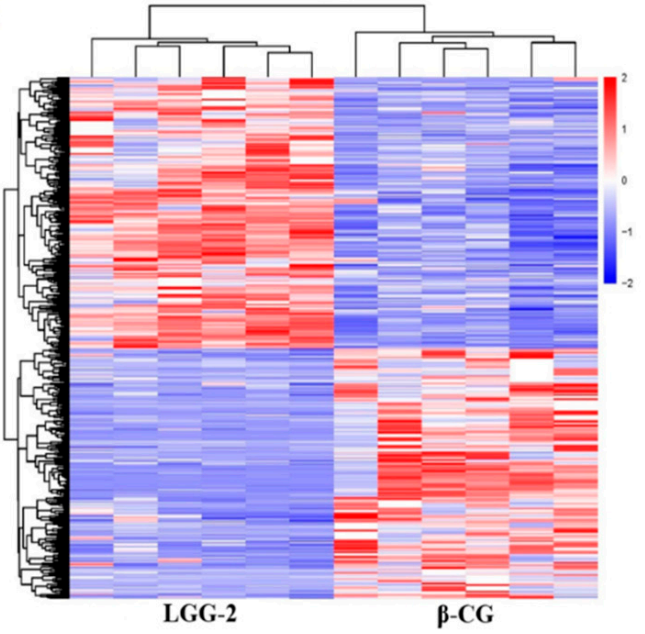

D
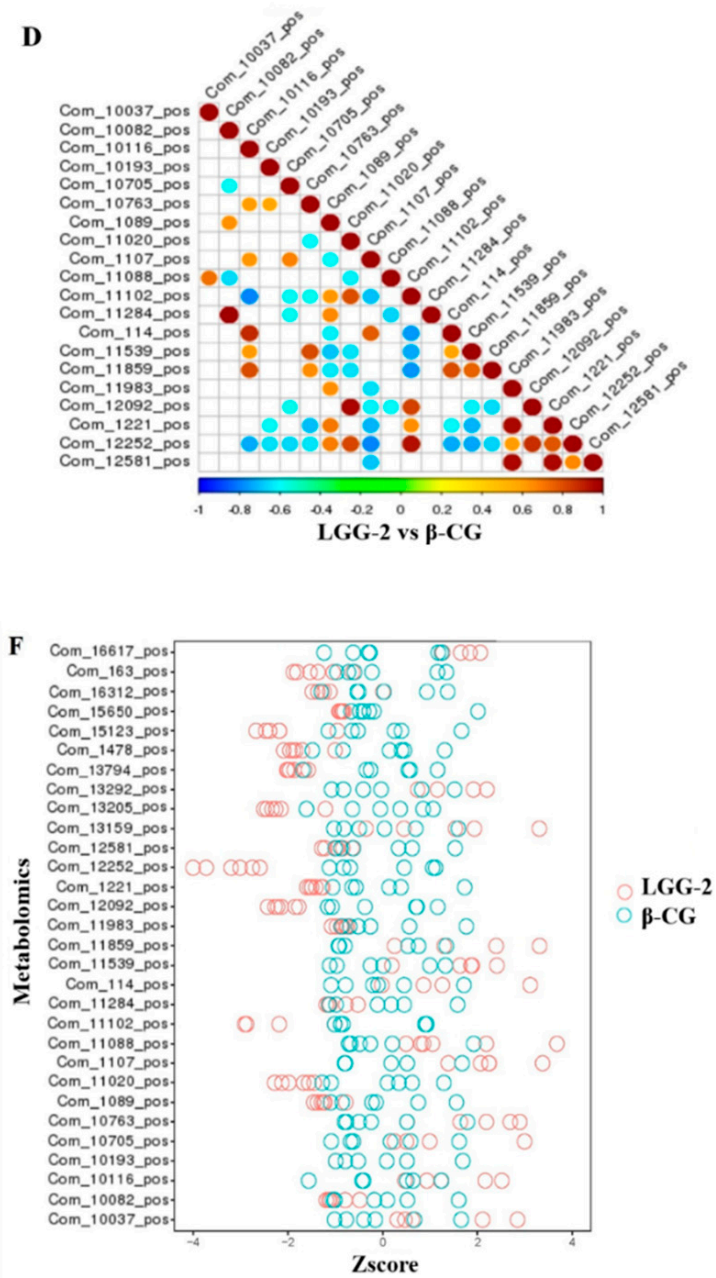

Figure 5. Analysis of differential metabolites between different groups. (A) Cluster heat map of differential metabolites in positive ion mode between the LGG-1 group and the NR-1 group. (B) Cluster heat map of differential metabolites in positive ion mode between the LGG-2 group and the $\beta$-CG group; (C) Correlation diagram of differential metabolites in positive ion mode between the LGG-1 group and the NR-1 group; (D) Correlation diagram of differential metabolites in positive ion mode between the LGG-2 group and the $\beta$-CG group; (E) Z-score chart in positive ion mode between the LGG-1 group and the NR-1 group; (F) Z-score chart in positive ion mode between the LGG-2 group and the $\beta$-CG group. 
A

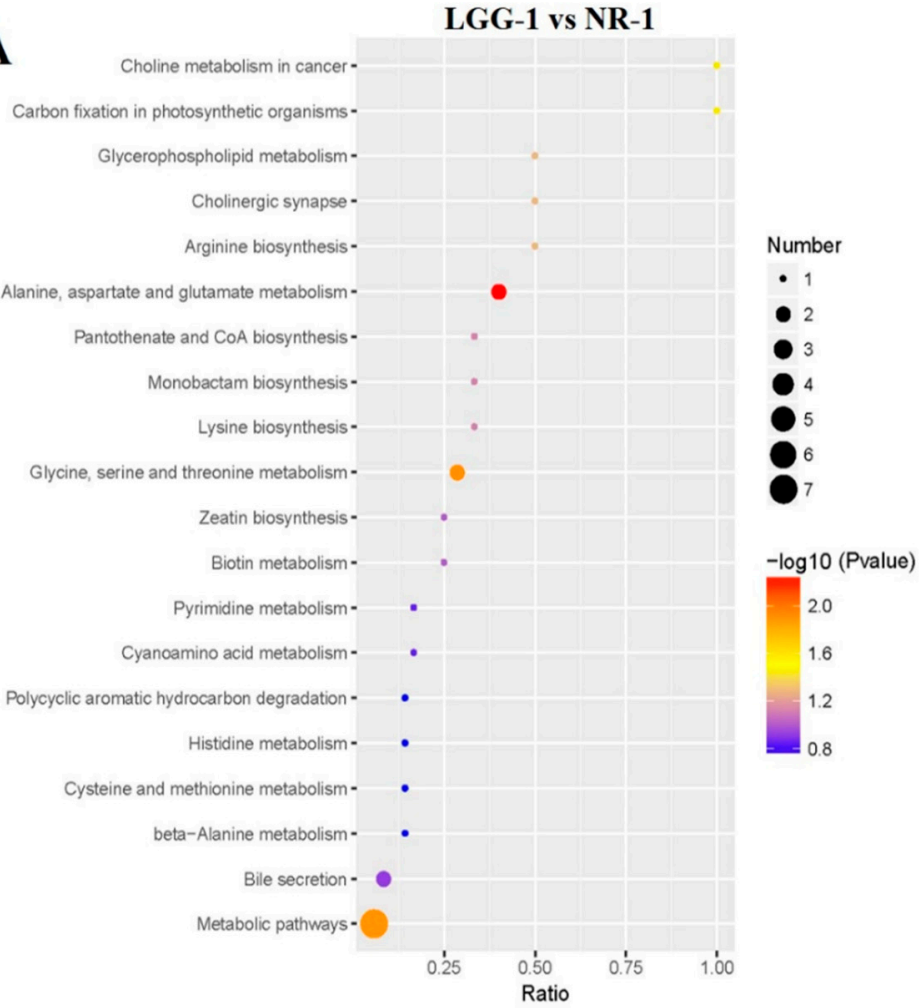

B

\section{LGG-2 vs $\beta$-CG}

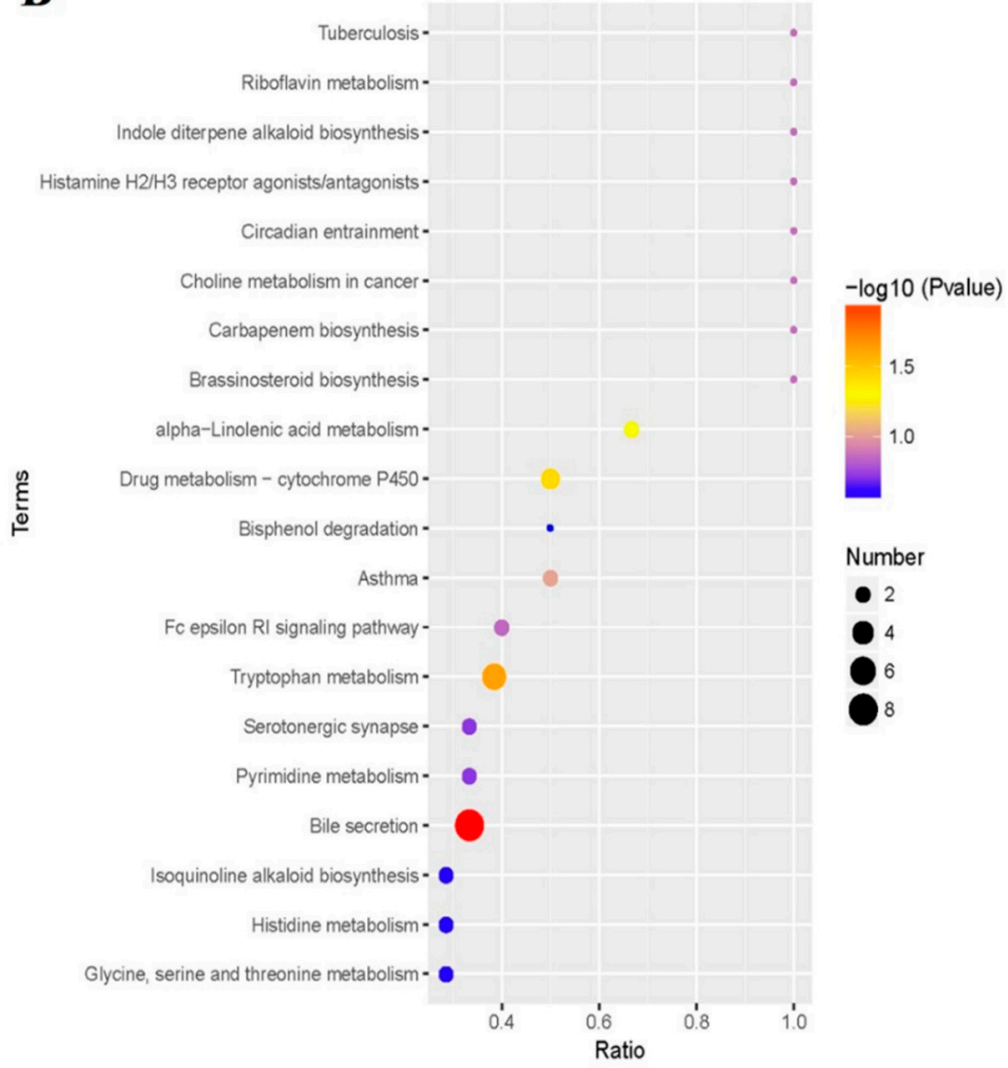

Figure 6. Comparing the bubble chart of the enriched KEGG pathway between different groups. (A) Metabolomic pathway chart in positive ion mode between the LGG-1 group and the NR-1 group; (B) Metabolomic pathway chart in positive ion mode between the LGG-2 group and the $\beta$-CG group. 


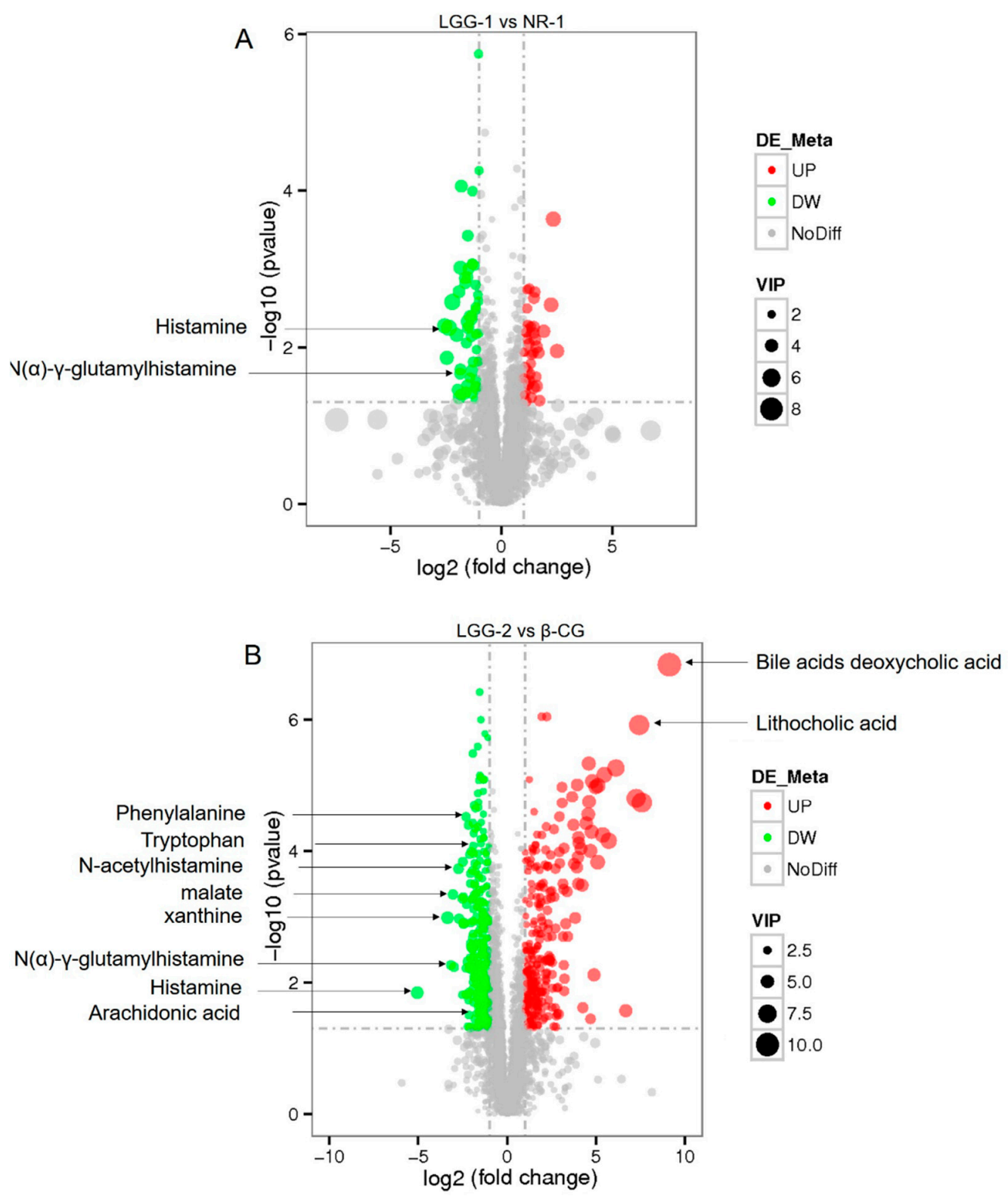

Figure 7. Identification of metabolites in the bile secretion pathway. (A) Volcano map of differential metabolites in positive ion mode between the LGG-1 group and the NR-1 group; (B) Volcano map of differential metabolites in positive ion mode between the LGG-2 group and the $\beta$-CG group.

\section{Discussion}

Apoptosis induced by allergies has recently received increased attention. Usually, the allergic response leads to intestinal damage, such as the rupture of intestinal villi, increased intestinal permeability, and increased numbers of apoptotic cells. In our previous work [22], we found that LGG alleviates $\beta$-CG allergy by modulating the differentiation of immune cells in the TCR signaling pathway. However, the effect of LGG on alleviating cell apoptosis has not been reported. LGG is recognized to regulate the gut microbiota and metabolites; therefore, in this study, we explored the effects of LGG intervention on cell apoptosis induced by $\beta-C G$ allergy and the related mechanisms and metabolites in gut microbiota.

Our experiments demonstrated that $\beta$-CG allergy induced cell apoptosis, including the occurrence of intestinal inflammation and the destruction of the intestinal wall barrier. Following LGG intervention, apoptotic cells were significantly decreased, and the state of intestinal villi was restored. It has been reported that the development of normal tolerance 
of apoptotic cells is related to pro-inflammatory bacteria [24,25]. Probiotics can reduce pro-inflammatory bacteria, and maintain intestinal immune homeostasis [26]. Therefore, LGG intervention may reduce the number of apoptotic cells by lowering the tolerance of apoptotic cells.

Probiotic-related immunomodulatory effects on the intestinal tract have been verified and reported. It has been reported that probiotic Enterococcus faecium has a regulatory effect on necrotic enteritis-induced intestinal barrier damage [27]. Probiotic Pediococcus acidilactici can restore intestinal morphology and enteric immunity [28]. LGG has been demonstrated to possess the recovery effect on intestinal inflammation induced by lipopolysaccharide [29]. It was observed that $\beta$-CG-induced allergy could lead to an increase in the number of apoptotic cells and rupture of intestinal villi in current study, whereas LGG intervention was verified to have the functionality to effectively reduce the number of apoptotic cells and restore the normal state of intestinal villi. The underlying mechanism may rely on the enhanced intestinal mucosal barrier function and intestinal immune response induced by LGG, which facilitated the recovery of the intestinal tissue damage. LGG has the capacity to inhibit cell apoptosis of the intestine, regulate homeostasis of intestinal epithelium, and prevent intestinal inflammatory diseases. On this basis, further research revealed that LGG's inhibition of cell apoptosis is related to the gut microbiota. The gut microbiota in healthy individuals is in a dynamic equilibrium state, whereas allergic mice were in an imbalanced state. From our experimental results, the relative abundance of Enterobacter increased in $\beta-C G$ allergic mice and then decreased after LGG intervention. According to reports, the imbalance of gut microbiota is directly related to allergies [30]. The microbial composition in the intestinal tract of normal mice is diverse, and the proportion of Enterobacter is low. Bridgman et al. reported changes in the gut microbiota induced by children's allergies, in which the abundance of Enterobacter increased [31]. Thus, $\beta-C G$ allergy induced an increase in Enterobacter, and LGG intervention reduced Enterobacter. The results of this study have showed that decreased Bacteroides in the intestine of allergic mice, which increased after LGG intervention. Bacteroides is the most common beneficial bacteria in the human ileum and large intestine, and the main component of the host's normal flora. Bacteroides has a symbiotic relationship with the host. Some studies have confirmed that intestinal bacteria, such as Bifidobacteria and Lactobacilli, are involved in reducing the risk of allergic diseases. Enterococcus and other allergic bacteria, such as Enterobacteriaceae and Clostridia, increase the risk of developing allergies [32]. Reddel et al. showed that probiotics promote the abundance of Bacteroides in the intestine of infants [33]. Therefore, the results revealed that LGG could restore normal microbiota, reduce pro-inflammatory bacteria, and regulate the imbalance in the gut microbiota.

The metabolic pathways of the gut microbiota are essential for maintaining intestinal immune homeostasis, and they affect the host's immunity to a variety of immune-mediated diseases. In the metabolic results, the bile secretion pathway was significantly enriched after LGG intervention. The metabolites produced by the bile secretion pathway have immunomodulatory functions [34]. Thus, we infer that LGG intervention could play an immunomodulatory role in the bile secretion pathway.

Future research needs to further identify the key metabolites in the bile secretion pathway because the mutual regulation between the host and its microbiome occurs through the secretion of metabolites. The bile secretion pathway can be achieved by regulating the metabolic products of the metabolic and inflammatory pathways. During $\beta-\mathrm{CG}$ allergy, histamine is present in relatively high concentrations in the bile secretion pathway. After LGG intervention, key differential metabolites regulate the immune responses, including upregulation of deoxycholic acid and cholic acid and downregulation of histamine in the bile secretion pathway. Recent studies have reported that metabolites play an essential role in the immune system [35]. Histamine is a biogenic amine that has a wide range of effects on many cell types, and the activation of receptors (H1R-H4R) mediates this effect, such as causing metabolic abnormalities and allergic diseases. The concentration of histamine depends on the expression and activity of histamine receptors [36]. Histamine metabolism 
is closely related to food allergy [37]. Deoxycholic acid and lithocholic acid play a conductive role in maintaining the homeostasis of the bile secretion pathway. Deoxycholic acid and lithocholic acid can activate FXR, after which FXR heterodimerizes with 9-cis retinoic $X$ receptor (RXR) to regulate cholesterol catabolism and BA biosynthesis [38]. Therefore, LGG intervention could regulate key differential metabolites in the bile secretion pathway.

The gut microbiota and its metabolites are closely related, and their interaction plays an important role in the immune system. Bacteroides express bile salt hydrolase (BSH), which deconjugates taurine-conjugated BAs and glycine-conjugated BAs. Metabolites may activate FXR in the intestine to regulate the bile secretion pathway and participate in the immune regulatory system. The role of the bile secretion pathway in the immune system is to increase the expression of $M u c 2$ in the intestine and induce production of the components of mucosal immune cells, thereby reducing apoptotic cells. In conclusion, we think that apoptotic cells in the intestine were decreased due to the increase in Bacteroides, which promoted the bile secretion pathway following LGG intervention (Figure 8).

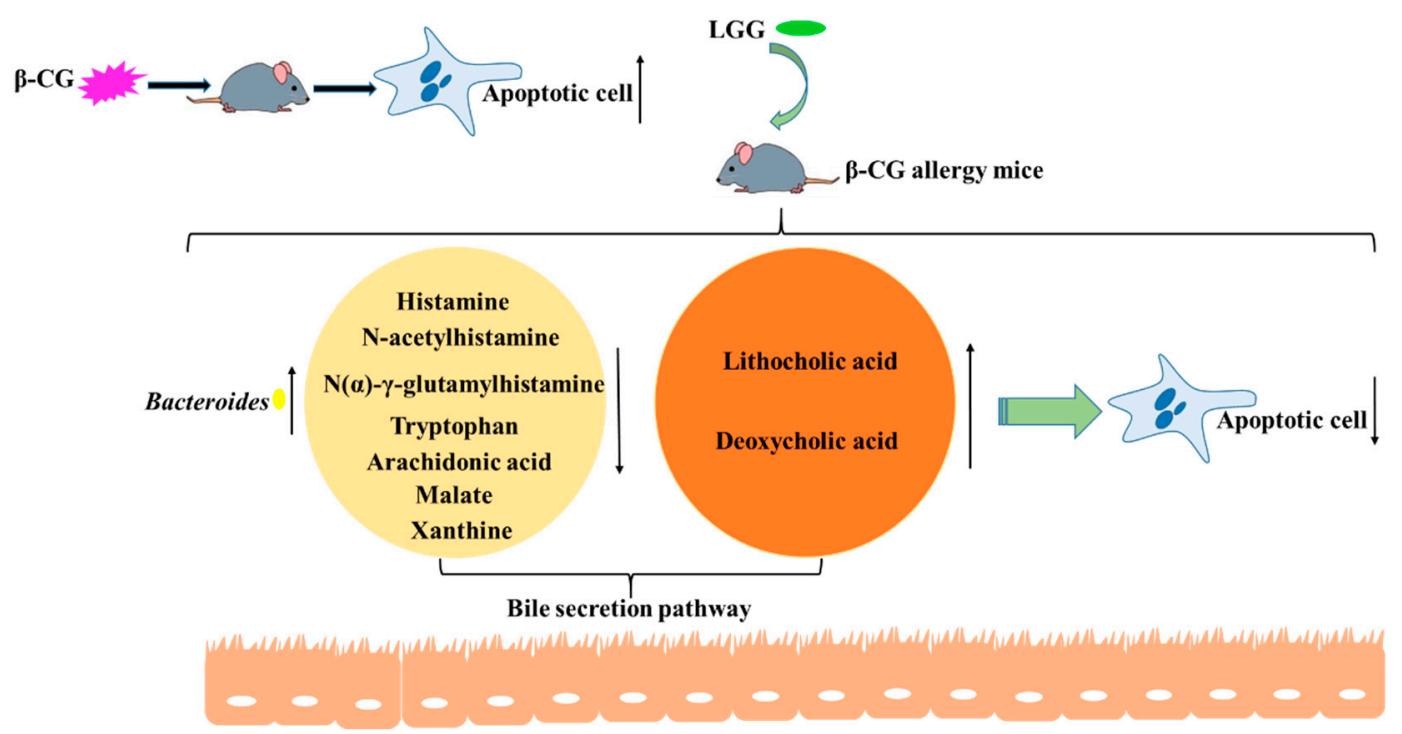

Figure 8. Mechanism of LGG reducing apoptotic cells through regulating Bacteroides levels and the bile secretion pathway.

\section{Conclusions}

In this study, we found through TUNEL analysis that LGG could reduce cell apoptosis induced by $\beta$-CG allergy. Further analysis of the gut microbiota and metabolic pathways of the intestinal contents revealed that LGG intervention regulated Bacteroides levels and the bile secretion pathway. As a new perspective for reducing apoptosis of cells, our findings provide a basis for the application of dietary intervention in the treatment of allergies.

Author Contributions: X.C. wrote the manuscript; Y.W. collected experimental data; Y.Z. and Y.H. participated in revising this article; Y.Z. and S.W. conceived and designed the experiments. All authors have read and agreed to the published version of the manuscript.

Funding: This work was supported by grants from the National Natural Science Foundation of China (No. 32072332).

Institutional Review Board Statement: Animal experiments were performed in accordance with the guidelines of the Institutional Animal Ethics Committee and were supported by the Institute of Radiation Medicine, Chinese Academy of Medical Sciences (IRM-DWLL-2020094).

Informed Consent Statement: Informed consent was obtained from all subjects involved in the study.

Data Availability Statement: The data presented in this study are available on request from the corresponding author. The data are not publicly available due to ethical and privacy reasons.

Conflicts of Interest: The authors declare no conflict of interest. 


\section{References}

1. $\mathrm{Bu}, \mathrm{G}$.; Li, T.; Zhao, Y.; Chen, F. Effects of high hydrostatic pressure combined with heat treatment on the antigenicity and conformation of $\beta$-conglycinin. Eur. Food Res. Technol. 2020, 246, 1065-1072. [CrossRef]

2. Ippoushi, K.; Wakagi, M.; Hashimoto, N.; Takano-Ishikawa, Y. Absolute quantification of the a, $\mathrm{a}^{\prime}$, and $\beta$ subunits of $\beta$-conglycinin from soybeans by liquid chromatography/tandem mass spectrometry using stable isotope-labelled peptides. Food Res. Int. 2019, 116, 1223-1228. [CrossRef] [PubMed]

3. Sastre, B.; Cañas, J.A.; Rodrigo-Muñoz, J.M.; Fernandez-Nieto, M.; Barranco, P.; Quirce, S.; Sastre, J.; Del Pozo, V. EosinophilDerived Exosomes Contribute to Asthma Remodeling by Activating Structural Lung Cells. Clin. Exp. Allergy 2018, 141 , AB72. [CrossRef]

4. Gao, H.; Feng, B.S.; Liu, J.Q.; Mo, L.H.; Geng, X.R.; Xiao, Y.; Zhang, Y.Y.; Hong, J.Y.; Liu, Z.J.; Liu, Z.G. Survivin induces defects in apoptosis in eosinophils in intestine with food allergy. Innate Immun. 2019, 25, 244-254. [CrossRef] [PubMed]

5. Wu, B.; Cui, H.; Peng, X.; Fang, J.; Zuo, Z.; Deng, J.; Huang, J. Dietary nickel chloride induces oxidative stress, apoptosis and alters Bax/Bcl-2 and caspase-3 mRNA expression in the cecal tonsil of broilers. Food Chem. Toxicol. 2014, 63, 18-29. [CrossRef] [PubMed]

6. Santos, F.D.S.; Mazzoli, A.; Maia, A.R.; Saggese, A.; Isticato, R.; Leite, F.; Iossa, S.; Ricca, E.; Baccigalupi, L. A probiotic treatment increases the immune response induced by the nasal delivery of spore-adsorbed TTFC. Microb. Cell Fact. 2020, 19, 1-13. [CrossRef]

7. Hamsah, H.; Widanarni, W.; Alimuddin, A.; Yuhana, M.; Junior, M.Z.; Hidayatullah, D. Immune response and resistance of Pacific white shrimp larvae administered probiotic, prebiotic, and synbiotic through the bio-encapsulation of Artemia sp. Aquac. Int. 2019, 27, 567-580. [CrossRef]

8. Maruščáková, I.C.; Schusterová, P.; Bielik, B.; Toporčák, J.; Mudroňová, D. Effect of Application of Probiotic Pollen Suspension on Immune Response and Gut Microbiota of Honey Bees (Apis mellifera). Probiotics Antimicrob. Proteins 2020, 12, 929-936. [CrossRef]

9. Ankita, S.; Sarangi, A.N.; Amit, G.; Rajni, S.; Rajat, B.; Priyanka, G.; Amita, A.; Rakesh, A. Effect of administration of a probiotic preparation on gut microbiota and immune response in healthy women in India: An open-label, single-arm pilot study. BMC Gastroenterol. 2018, 18, 85.

10. Ramírez, C.; Rojas, R.; Romero, J. Partial Evaluation of Autochthonous Probiotic Potential of the Gut Microbiota of Seriola lalandi. Probiotics Antimicrob. Proteins 2020, 12, 672-682. [CrossRef]

11. Ma, J.; Zhang, J.; Li, Q.; Shi, Z.; Wu, H.; Zhang, H.; Tang, L.; Yi, R.; Su, H.; Sun, X. Oral administration of a mixture of probiotics protects against food allergy via induction of CD103+ dendritic cells and modulates the intestinal microbiota. J. Funct. Foods 2019, 55, 65-75. [CrossRef]

12. Nie, Y.F.; Yan, X.H. Cross-talk between bile acids and intestinal microbiota in host metabolism and health. J. Zhejiang Univ. Sci. B 2015, 16, 436-446. [CrossRef] [PubMed]

13. Wahlstrom, A.; Sayin, S.I.; Marschall, H.; Backhed, F. Intestinal Crosstalk between Bile Acids and Microbiota and Its Impact on Host Metabolism. Cell Metab. 2016, 24, 41-50. [CrossRef] [PubMed]

14. Enright, E.F.; Griffin, B.T.; Gahan, C.G.M.; Joyce, S.A. Microbiome-mediated bile acid modification: Role in intestinal drug absorption and metabolism. Pharmacol. Res. 2018, 133, 170-186. [CrossRef] [PubMed]

15. Dawson, P.A.; Shneider, B.L.; Hofmann, A.F. Bile formation and the enterohepatic circulation. In Physiology of the Gastrointestinal Tract; Elsevier: Amsterdam, The Netherlands, 2018; pp. 931-956.

16. Duboc, H.; Rajca, S.; Rainteau, D.; Benarous, D.; Maubert, M.; Quervain, E.; Thomas, G.; Barbu, V.; Humbert, L.; Despras, G. Connecting dysbiosis, bile-acid dysmetabolism and gut inflammation in inflammatory bowel diseases. Gut 2013, 62, 531-539. [CrossRef]

17. Irina, S.; Petrova, M.I.; Astrid, F.; Lore, P.; Jeroen, V.; Ceuppens, J.L.; Sven, S.; Sarah, L. Intranasal administration of probiotic Lactobacillus rhamnosus GG prevents birch pollen-induced allergic asthma in a murine model. Allergy 2019, 74, 100-110.

18. Yazdi, F.G.; Zakeri, A.; Ark, I.V.; Leusink-Muis, T.; Folkerts, G. Crude Turmeric Extract Improves the Suppressive Effects of Lactobacillus rhamnosus GG on Allergic Inflammation in a Murine Model of House Dust Mite-Induced Asthma. Front. Immunol. 2020, 11, 1092. [CrossRef]

19. Juan, Z.; Jing-Yi, M.; Qiu-Hong, L.; Hui, S.; Xin, S. Lactobacillus rhamnosus GG induced protective effect on allergic airway inflammation is associated with gut microbiota. Cell. Immunol. 2018, 332, 77-84.

20. Basturk, A.; Isik, İ.; Atalay, A.; Y1lmaz, A. Investigation of the Efficacy of Lactobacillus rhamnosus GG in Infants with Cow's Milk Protein Allergy: A Randomised Double-Blind Placebo-Controlled Trial. Probiotics Antimicrob. Proteins 2020, 12, 138-143. [CrossRef]

21. Guadamuro, L.; Diaz, M.; Jiménez, S.; Molinos-Norniella, C.; Pérez-Solis, D.; Rodríguez, J.M.; Bousoo, C.; Gueimonde, M.; Margolles, A.; Delgado, S. Fecal Changes following Introduction of Milk in Infants with Outgrowing Non-IgE Cow's Milk Protein Allergy Are Influenced by Previous Consumption of the Probiotic LGG. Front. Immunol. 2019, 10, 1819. [CrossRef]

22. Chen, X.; Zhao, X.; Hu, Y.; Zhang, B.; Zhang, Y.; Wang, S. Lactobacillus rhamnosus GG alleviates $\beta$-conglycinin induced allergy by regulating the $\mathrm{T}$ cell receptor signalling pathway. Food Funct. 2020, 11, 10554-10567. [CrossRef] [PubMed]

23. Ippoushi, K.; Tanaka, Y.; Wakagi, M.; Hashimoto, N. Evaluation of protein extraction methods for $\beta$-conglycinin quantification in soybeans and soybean products. LWT Food Sci. Technol. 2020, 132, 109871. [CrossRef]

24. Mendoza-Reinoso, V.; Baek, D.Y.; Kurutz, A.; Rubin, J.R.; Koh, A.J.; Mccauley, L.K.; Roca, H. Unique Pro-Inflammatory Response of Macrophages during Apoptotic Cancer Cell Clearance. Cells 2020, 9, 429. [CrossRef] [PubMed] 
25. Welcome, M.O. Gut Microbiota Disorder, Gut Epithelial and Blood-Brain Barrier Dysfunctions in Etiopathogenesis of Dementia: Molecular Mechanisms and Signaling Pathways. Neuromol. Med. 2019, 21, 205-226. [CrossRef]

26. Wang, H.; Gao, K.; Wen, K.; Allen, I.C.; Li, G.; Zhang, W.; Kocher, J.; Yang, X.; Giri-Rachman, E.; Li, G.-H.; et al. Lactobacillus rhamnosus GG modulates innate signaling pathway and cytokine responses to rotavirus vaccine in intestinal mononuclear cells of gnotobiotic pigs transplanted with human gut microbiota. BMC Microbiol. 2016, 16, 109. [CrossRef]

27. Wu, Y.; Zhen, W.; Geng, Y.; Wang, Z.; Guo, Y. Pretreatment with probiotic Enterococcus faecium NCIMB 11181 ameliorates necrotic enteritis-induced intestinal barrier injury in broiler chickens. Sci. Rep. 2019, 9, 1-17. [CrossRef]

28. Al-Hisnawi, A.; Rodiles, A.; Rawling, M.D.; Castex, M.; Waines, P.; Gioacchini, G.; Carnevali, O.; Merrifield, D.L. Dietary probiotic Pediococcus acidilactici MA18/5M modulates the intestinal microbiota and stimulates intestinal immunity in rainbow trout (Oncorhynchus mykiss). J. World Aquac. Soc. 2019, 50, 1133-1151. [CrossRef]

29. Li, X.; Hu, D.; Tian, Y.; Song, Y.; Hou, Y.; Sun, L.; Zhang, Y.; Man, C.; Zhang, W.; Jiang, Y. Protective effects of a novel Lactobacillus rhamnosus strain with probiotic characteristics against lipopolysaccharide-induced intestinal inflammation in vitro and in vivo. Food Funct. 2020, 11, 5799-5814. [CrossRef]

30. Cukrowska, B.; Biera, J.B.; Zakrzewska, M.; Klukowski, M.; Maciorkowska, E. The Relationship between the Infant Gut Microbiota and Allergy. The Role of Bifidobacterium breve and Prebiotic Oligosaccharides in the Activation of Anti-Allergic Mechanisms in Early Life. Nutrients 2020, 12, 946. [CrossRef]

31. Bridgman, S.L.; Kozyrskyj, A.L.; Scott, J.A.; Becker, A.B.; Azad, M.B. Gut microbiota and allergic disease in children. Ann. Allergy Asthma Immunol. 2016, 116, 99-105. [CrossRef]

32. Reddel, S.; Mennini, M.; Del Chierico, F.; Vernocchi, P.; Valluzzi, R.; Fierro, V.; Riccardi, C.; Fiocchi, A.; Putignani, L. Gut microbiota profile in infants with milk and/or egg allergy and evaluation of intestinal colonization and persistence of a probiotic mixture. World Allergy Organ. J. 2020, 13, 100424. [CrossRef]

33. Rooks, M.G.; Garrett, W.S. Gut microbiota, metabolites and host immunity. Nat. Rev. Immunol. 2016, 16, 341-352. [CrossRef] [PubMed]

34. Kim, C.H. Immune regulation by microbiome metabolites. Immunology 2018, 154, 220-229. [CrossRef] [PubMed]

35. Levy, M.; Blacher, E.; Elinav, E. Microbiome, metabolites and host immunity. Curr. Opin. Microbiol. 2017, 35, 8-15. [CrossRef]

36. Smolinska, S.; Jutel, M.; Crameri, R.; Omahony, L. Histamine and gut mucosal immune regulation. Allergy 2014, 69, 273-281. [CrossRef]

37. Amo, G.; Cornejo-García, J.A.; García-Menaya, J.M.; Cordobes, C.; Torres, M.J.; Esguevillas, G.; Mayorga, C.; Martinez, C.; Blanca-Lopez, N.; Canto, G.; et al. FCERI and Histamine Metabolism Gene Variability in Selective Responders to NSAIDS. Front. Pharmacol. 2016, 7, 353. [CrossRef]

38. Byun, S.; Kim, D.-H.; Ryerson, D.; Kim, Y.-C.; Sun, H.; Kong, B.; Yau, P.; Guo, G.; Xu, H.E.; Kemper, B. Postprandial FGF19-induced phosphorylation by Src is critical for FXR function in bile acid homeostasis. Nat. Commun. 2018, 9, 1-14. [CrossRef] 